TecnoHumanismo. Revista Científica

Diciembre 2021 - Febrero 2022

Volumen 1 / No.4

ISSN: 2710-2394

pp. 244-253

HUMANISMO

https://doi.org/10.53673/th.v2i2.107

https://tecnohumanismo.online

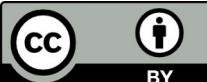

\title{
Blockchain y la innovación en las tecnologías
}

\author{
Blockchain and innovation in technologies \\ Blockchain e inovação em tecnologias
}

ARTÍCULO GENERAL

\section{Wilfredo Zevallos Umpiri \\ wizeuce@gmail.com}

https://orcid.org/0000-0001-5915-7852

Investigador Independiente, Lima - Perú

Recibido 07 de Junio 2021 | Arbitrado y aceptado 25 de Agosto 2021 | Publicado en 04 Diciembre 2021

\begin{abstract}
RESUMEN
El presente artículo se propuso como objetivo general reflexionar sobre Blockchain y sus vínculos con la innovación en las tecnologías. Ello en la medida que la cadena de bloques hace posible que existan innovaciones de productos y procesos, que se infiere tienen grandes beneficios en diversos sectores de la vida de los seres humano. Para ello, se aplicó una metodología basada en una revisión bibliográfica de tipo descriptiva, utilizando artículos científicos como fuentes primarias de información. La variable u objeto de estudio seleccionado fue Blockchain. Se hizo la búsqueda a través de Web o Science, Proquest, Scielo y Scopus. Los criterios fueron: 1) artículos que incluyeran las palabras "Blockchain" en el título, resumen o palabras claves; 2) que se apoyen con palabras clave complementarias como "innovación" y "tecnología" y, 3) artículos publicados entre el año 2016 y julio del 2021. Se seleccionaron artículos de América Latina, Norteamérica y Europa. Se organizaron los resultados y discusión por temáticas que recalcan los principales hallazgos. Durante todo el estudio y recopilación de documentación se pudo concluir que existe en la literatura gran interés por el aprovechamiento del Blockchain, vinculando a esta tecnología con la innovación en diversos aspectos de la vida humana (economía y finanzas, gestión pública y temas legales, salud y sociedad). En ese sentido, se concluyó con el objetivo general reflexionar sobre Blockchain y sus vínculos con la innovación en las tecnologías.

Palabras clave: Blockchain; innovación; tecnologías
\end{abstract}

\begin{abstract}
The general objective of this article was to reflect on Blockchain and its links with innovation in technologies. This to the extent that the blockchain makes possible product and process innovations, which are inferred to have great benefits in various sectors of human life For this, a methodology based on a descriptive literature review was applied, using scientific articles as primary sources of information. The selected variable or object of study was Blockchain. The search was made through Web or Science, Proquest, Scielo and Scopus. The criteria were: 1) articles that included the words "Blockchain" in the title, abstract or keywords; 2) that are supported by complementary keywords such as "innovation" and "technology" and, 3) articles published between 2016 and July 2021. Articles from Latin America, North America and Europe were selected. The results and discussion were organized by themes that emphasize the main findings. Throughout the study and compilation of documentation, it was possible to conclude that there is great interest in the literature in the use of Blockchain, linking this technology with innovation in various aspects of human life (economy and finance, public management and legal issues, health and society). In this sense, it was concluded with the general objective to reflect on Blockchain and its links with innovation in technologies.

Keywords: Blockchain; innovation; technologies
\end{abstract}

\section{RESUMO}

O objetivo geral deste artigo foi refletir sobre Blockchain e suas ligações com a inovação em tecnologias. Isso na medida em que o blockchain possibilita inovações de produtos e processos, que se infere de grandes benefícios em diversos setores da vida humana. Para isso, foi aplicada uma metodologia baseada em uma revisão descritiva da literatura, utilizando artigos científicos como fontes primárias de informação. A variável ou objeto de estudo selecionado foi Blockchain. A busca foi feita através da Web ou Science, Proquest, Scielo e Scopus. Os critérios foram: 1) artigos que incluíssem as palavras "Blockchain" no título, resumo ou palavras-chave; 2) que são apoiados por palavras-chave complementares como "inovação" e "tecnologia" e, 3) artigos publicados entre 2016 e julho de 2021. Foram selecionados artigos da América Latina, América do Norte e Europa. Os resultados e a discussão foram organizados por temas que enfatizam os principais achados. Ao longo do estudo e compilação da documentação, foi possível concluir que existe um grande interesse da literatura na utilização do Blockchain, relacionando esta tecnologia com a inovação em vários aspetos da vida humana (economia e finanças, gestão pública e questões jurídicas, saúde e sociedade). Nesse sentido, concluiu-se com o objetivo geral de refletir sobre Blockchain e suas ligações com a inovação em tecnologias.

Palavras-chave: Blockchain; inovação; tecnologias 


\section{Introducción}

Los conceptos de bitcoin y blockchain los propuso por vez primera vez en el 2008 alguien que usaba el seudónimo de Satoshi Nakamoto, quien también describió cómo la criptología y un libro mayor distribuido abierto pueden combinarse en una aplicación de moneda digital (Nakamoto, 2008). Tras escribir el artículo e intercambiar mails durante dos años con colegas sobre todos los aspectos posibles por analizar respecto a la moneda virtual, Nakamoto desapareció en 2011 (BBVA Innovation Center, 2016).

Blockchain, también denominado cadena de bloques, es en la actualidad un concepto extenso utilizado para la representación de todo un nuevo conjunto de tecnologías. Debido a su novedad, hay gran confusión respecto a su definición exacta (Arrasco, 2021). No obstante (Dolader et al., 2017) la conceptualiza, de forma general, como una base de datos la cual puede compartirse entre un gran número de usuarios en forma peer-to-peer. Además, permite que se almacene información de forma ordenada y sin que nunca cambie. Cabe mencionar que, solo si existe un acuerdo entre la mayoría de las partes que intervienen, podrá adjuntarse la información a la cadena de bloques.

Es debido a los nodos o «mineros» que se crean nuevos bloques. Estos mineros son nodos de la red que forman parte del proceso de escritura de datos en la blockchain a cambio de una recompensa económica. La misma fuente señala, además, que el proceso que hace posible que se desarrolle un consenso garantizado entre los mineros de la cadena de bloques para el orden de escritura de estos es lo que se ha denominado Proof-of-work (PoW).

Entonces, (Arrasco, 2021) resume Blockchain, señalando que es una cadena de bloques informáticos, donde cada bloque contiene información de todo tipo (liquidez, derechos, propiedades, certificados, entre otros.), y estos se van añadiendo uno tras otro agrandando la cadena. Una vez que los datos se introdujeron en la cadena no pueden ser modificados; esto permite que se pueda verificar en el tiempo diferentes transacciones y genera seguridad y confianza en los datos almacenados. Dicha seguridad y confianza hace posible que se pueda realizar diversas acciones sin que se necesite a un intermediario (por ejemplo, un banco) lo que disminuye también el coste de transacción.

Por todo ello, la cadena de bloques hace posible que existan cadenas de valor más ágiles, innovaciones de productos más rápidas, relaciones más estrechas con los clientes y una integración más rápida con IoT y la tecnología en la nube (Ahram et al., 2017). 
Además, que se lleve el alcance de la innovación a diferentes tipos de aplicaciones en diversos sectores de la sociedad (Arrasco, 2021). En ese sentido, la presente se propone como objetivo general reflexionar sobre Blockchain y sus vínculos con la innovación en las tecnologías.

\section{Metodología}

La investigación se basó en una revisión bibliográfica de tipo descriptiva, utilizando artículos científicos como fuentes primarias de información. La variable u objeto de estudio seleccionado fue Blockchain. La revisión bibliográfica se llevó a cabo en las bases de datos Web o Science, Proquest, Scielo y Scopus. La selección de estos fue a partir de los siguientes criterios: 1) artículos que incluyeran las palabras "Blockchain" en el título, resumen o palabras claves; 2) que se apoyen con palabras clave complementarias como “innovación” y “tecnología” y, 3) artículos publicados entre el año 2016 y julio del 2021.

Tras realizar la búsqueda de documentos, se seleccionó un promedio de 30 artículos. Con ello se pudo realizar una lectura meticulosa de cada uno de ellos para comprobar la presencia de las palabras "Blockchain" "innovación" y "tecnología” en sus títulos, resúmenes o palabras claves. A través de esta técnica se descartaron diez artículos que no contenían dichas palabras en las secciones mencionadas o que no pertenecían al periodo de tiempo seleccionado.

Según estos criterios fueron seleccionados artículos de América Latina, Norteamérica y Europa. Se organizaron los resultados y discusión por temáticas que recalcan los principales hallazgos con la información detallada y plasmada de forma concisa.

\section{Resultados y discusión}

Actualmente, se tienen cuatro tendencias importantes en el desarrollo de las soluciones a través del uso Blockchain durante los últimos cinco años, en primer lugar, se destaca la actividad inventiva y de patentamiento en el cual está el uso de Blockchain para el aseguramiento de comunicaciones y equipamiento electrónico; Blockchain en sistemas de pagos y comercio electrónico; Blockchain en sistemas de gestión de procesos empresariales en diferentes ámbitos; y por último, las tecnologías relacionadas con identidad digital y registros públicos (Olivero et al., 2020). 
Figura 1. Tendencias tecnológicas según la actividad inventiva y de patentamiento



Fuente. (Olivero et al., 2020).

Pero, además, lo que ha supuesto Blockchain en la innovación se expande a otros campos. Así, se ha podido observar en la literatura cómo ha permitido esta tecnología que se hagan renovaciones e invenciones en sectores económicos, legales, y sociales.

\section{Aplicaciones económicas}

Las empresas pueden aprovechar las cadenas de bloques de diversas formas para obtener una ventaja sobre sus competidores. Pueden optimizar su negocio principal, reducir los costos de transacción y hacer que la propiedad intelectual y los pagos sean más transparentes y automatizados (Xu et al., 2019). Semejante postura toma (Lewis et al., 2017) quien señala que Blockchain tiene el potencial de proporcionar grandes ganancias de eficiencia en empresas que actualmente requieren una intermediación costosa, incluidos los servicios financieros. Y aunque aún queda mucho trabajo por hacer, blockchain representa una fuente prometedora de innovación futura en los mercados financieros. 
En el comercio internacional, por ejemplo, China fue el país líder con 1.353 invenciones que corresponde al 40\%, le sigue Estados Unidos, con el 27\% y Japón con el $5 \%$ de participación. Por su parte, Latinoamérica por ser una región emergente en el desarrollo de este tipo de tecnologías presentó solo el 1\% de la producción mundial de patentes (Olivero et al., 2020).

También se ha encontrado en la literatura la búsqueda de nuevas soluciones sociotecnológicas que quieren dar una respuesta más adecuada al nuevo paradigma social, marcado por la constante innovación y evolución tecnológica. Ante los sistemas tradicionales del Estado y las entidades privadas, caracterizados por la primacía del capital, surge la Economía Social como mecanismo para satisfacer las necesidades humanas. Un sistema socio-económico determinado por un conjunto de actividades llevadas a cabo por entidades, para mejorar la situación de la sociedad de manera independiente respecto a los poderes públicos (Gallego \& Palomo, 2020). Vinculado a este nuevo panorama social, tecnológicamente se produjo una revolución del internet de la información al nuevo internet del valor o blockchain. Una nueva tecnología capaz de solventar los problemas actuales basándose en la descentralización de las autoridades gubernamentales a favor de una mayor confianza y autonomía social.

En la medida en la que la estructura tecnología del blockchain esté compuesta por un sistema participativo democráticamente, comprometido con el entorno y la sociedad y emplee bases de datos públicas, abiertas, anónimas o pseudoanómicas, así como sistemas de trabajo PoS, se alinearán en la consecución de los pilares de la Economía Social.

Algunas otras implicancias de Blockchain con las finanzas según (Gallego \& Palomo, 2020) son:

- El empleo de criptomonedas, criptodivisas, criptoactivos, o tokens que permiten una mayor participación global a colectivos excluidos.

Ello también lo sostiene (Chen, 2018) quien explica que los tokens de blockchain pueden democratizar el espíritu empresarial al brindarles a los emprendedores nuevas formas de recaudar fondos e involucrar a las partes interesadas, y la innovación al brindarles a los innovadores una nueva forma de desarrollar, implementar y difundir aplicaciones descentralizadas. 
- El empleo de la tecnología blockchain de un sistema de rastreo inmutable e inalterable. Este sistema mejora los sistemas de financiación basados en crowdfunding o microaportaciones.

- El uso de los Smart contracts que permite reducir costes al evitar el empleo de agentes intermedios, agilizar los trámites y aportar mayor seguridad ante posibles fallos humanos.

Acorde con (Olivero et al., 2020) la tecnología Blockchain pretende seguir impactando en los próximos años, buscando que su utilización sea masiva, pero no solamente en el sector financiero, sino también en otros sectores. Por ejemplo, en la protección de datos, el sector de la salud y estatal, entre otras. Esta tecnología podría cambiar el estilo de vida de la sociedad, desde el punto de vista del impulso de la innovación, seguridad en los sistemas informáticos y tratamientos de datos, cadenas de suministro, etc.

\section{Innovaciones Legales y de Gestión Pública}

(Blemus, 2018) expone un documento que recoge los últimos proyectos regulatorios principales y las consultas de toda la industria en los Estados Unidos, en la Unión Europea (UE) y en los principales países económicos donde se han discutido las regulaciones de Blockchain como propuesta y adopción.

En solo unos años, Blockchain se ha convertido en un tema importante para los tomadores de decisiones públicas en todo el mundo. Dado que esta tecnología disruptiva y descentralizada se ha convertido en un problema empresarial clave para las empresas emergentes y los participantes del mercado.

En ese sentido, el autor explica que tanto bancos centrales como los reguladores financieros han cambiado, sobre todo en los EE. UU y en la UE, de una fuerte hostilidad inicial a una más cautelosa y cautelosa posición favorable al mercado.

(Legerén, 2019) también razona esta tendencia pero en Europa y sostiene que, aunque sean muchos los proyectos que intentan utilizar la cadena de bloques no serán tantos los que finalmente cristalicen y se consoliden. Ello se debe, entre otros motivos, a que el resultado que han de arrojar habrá de -eliminando los «intermediarios innecesarios»)- satisfacer las necesidades existentes con el mismo grado de seguridad y a un menor costo; requisitos no sencillos de cumplir. En tal sentido, sí parece que reúne tales características y arraiga el uso de blockchain como plataforma para la realización de 
pagos -utilizando para ello smart contracts-; aspecto que -se reconozca o no en el futuro a las criptomonedas como dinero legal y no sólo como medio de pago-mejorará en cuanto se atempere la volatilidad de aquellas, propiciada, en buena medida, por la actividad especulativa.

Sea ello como fuere, en cualquier caso, se indica que las cadenas de bloques no reemplazarán ni la función notarial ni los Registros de derechos, tal y como están concebidos en Europa. Ninguno de ellos constituye un intermediario «innecesario». En efecto, ambos aportan valor con el control jurídico y de legalidad que realizan respecto de la transacción que se pretende efectuar, con el análisis de la capacidad o el consentimiento de las partes, o, en fin, porque acreditan el reconocimiento por parte del Estado de la titularidad de los derechos registrados, generando confianza en los operadores económicos.

\section{Sector salud}

Blockchain permite cadenas de valor más ágiles, innovaciones de productos más rápidas, relaciones más estrechas con los clientes y una integración más rápida con el Internet de las cosas (IoT) y la tecnología en la nube. Ahora, con las tecnologías Cloud y Blockchain que brindan alta potencia informática y capacidades de red, los sistemas cognitivos son herramientas disponibles para profundizar la relación entre los humanos y el mundo.

(Daniel et al., 2017) señala, por ejemplo, la unión de Blockchain con la computación móvil implementada en la red basada en Pervasive Social Network (PSN) mejorando la transferencia inalámbrica y la organización de registros médicos. Ello supone un aporte importante respecto los datos de seguimiento clínico. En ese sentido, se menciona que más de la mitad de los ensayos clínicos carecen de la disponibilidad de todos los métodos y resultados correspondientes. El protocolo sugerido en manos del contrato inteligente Blockchain con su confianza y transparencia adjuntas podría ser donde el nivel de servicio médico se puede mejorar significativamente.

Además, el uso de blockchain para almacenar registros médicos tiene el potencial de hacer que los datos privados de atención médica sean más seguros, escalables y a prueba de manipulaciones. La naturaleza distribuida de la cadena de bloques puede facilitar el intercambio de datos entre las partes autorizadas y salvar los silos de datos tradicionales, aumentando drásticamente la eficiencia y mejorando la coordinación de la 
atención. Los costos de la atención médica se pueden reducir mediante una mejor coordinación de las reclamaciones de seguros con el tratamiento prestado. La auditoría de datos se mejora a través de los registros inmutables mantenidos por blockchain. Los costos asociados con la minería de blockchain incluso se pueden compensar ofreciendo recompensas de metadatos anónimos (Heston, 2017).

\section{Conclusiones}

Durante todo el estudio y recopilación de documentación se pudo concluir que existe en la literatura gran interés por el aprovechamiento del Blockchain, vinculando a esta tecnología con la innovación en diversos aspectos de la vida humana (economía y finanzas, gestión pública y temas legales, salud y sociedad). En ese sentido, se cumplió con el objetivo general reflexionar sobre Blockchain y sus vínculos con la innovación en las tecnologías.

Además, se vio en la economía que ya se han iniciado cambios e inversiones como en la facturación electrónica, tokens, seguridad e identidad, así como en temas de gestión pública y de legalidad, donde se ha apreciado el trabajo de entidades internacionales así como de gobiernos de diversos países. También en los temas de legislación donde se aprecia un arduo trabajo para ponerse a la par de las innovaciones tecnológicos. Y, finalmente, en aspectos sociales, siendo el más relevante el de la salud, donde en varios países ya hay progresos al respecto y estos tienen que ver con temas administrativos mejorando sus gestiones y beneficiando en última instancia al ciudadano común.

\section{Referencias}

Ahram, T., Sargolzaei, A., Sargolzaei, S., Daniels, J., \& Amaba, B. (2017). Blockchain technology innovations. 2017 IEEE Technology and Engineering Management Society Conference, TEMSCON 2017, 2016, 137-141. https://doi.org/10.1109/TEMSCON.2017.7998367

Arrasco, H. (2021). Tecnologías Blockchain: Innovación Empresarial y Aplicación. https://upcommons.upc.edu/handle/2117/342840

BBVA Innovation Center. (2016). Tecnología Blockchain: el avance de bitcoin y de los pagos virtuales. https:/www.bbva.com/wp-content/uploads/2017/10/ebook-cibbvtecnologia_blockchain-es.pdf 
Blemus, S. (2018). Law and Blockchain: A Legal Perspective on Current Regulatory Trends Worldwide. SSRN Electronic Journal, 1-15. https://doi.org/10.2139/ssrn.3080639

Chen, Y. (2018). Blockchain tokens and the potential democratization of entrepreneurship and innovation. Business Horizons, 61(4), 567-575. https://doi.org/10.1016/j.bushor.2018.03.006

Daniel, J., Sargolzaei, A., Abdelghani, M., Sargolzaei, S., \& Amaba, B. (2017). Blockchain Technology, Cognitive Computing, and Healthcare Innovations. Journal of Advances in Information Technology, 8(3), 194-198. https://doi.org/10.12720/jait.8.3.194-198

Dolader, C., Bel, J., \& Muñoz, J. (2017). La blockchain: fundamentos, aplicaciones y relación con otras tecnologías disruptivas. Economía Industrial, 405, 33-40.

Gallego, B., \& Palomo, R. (2020). Blockchain: un reto del siglo XXI para la Economía Social. http://ciriec.es/wp-content/uploads/2020/09/COMUN-046-T11GALLEGO-PALOMO-ok.pdf

Heston, T. (2017). A case study in blockchain healthcare innovation. International Journal of Current Research, 9(11), 60587-60588. https://papers.ssrn.com/sol3/papers.cfm?abstract_id=3077455

Legerén, A. (2019). Retos jurídicos que plantea la tecnología de la cadena de bloques. Aspectos legales de blockchain. Revista de Derecho Civil, VI, 177-237.

Lewis, R., McPartland, J., Ranjan, R., Lewis, R., \& McPartland, J. (2017). Blockchain and Financial Market Innovation. Economic Perspectives, 7, 2-12.

Nakamoto, S. (2008). Bitcoin: A Peer-to-Peer Electronic Cash System. Artificial Life. https://doi.org/10.1162/ARTL_a_00247

Olivero, E., Vega, Y., Ávila, J., \& Peralta, P. (2020). Gestión de la innovación mediante la tecnología blockchain en el desarrollo del comercio internacional. In Impacto de la logística e innovación en el comercio internacional (pp. 103-118). Universidad Simón Bolívar.

Xu, M., Chen, X., \& Kou, G. (2019). A systematic review of blockchain. Financial Innovation, 5(1). https://doi.org/10.1186/s40854-019-0147-z 